\title{
Chaotic Properties of the Elliptical Stadium
}

\author{
Roberto Markarian* \\ Inst. de Matemática y Estadística "Prof. Ing. Rafael Laguardia" \\ Fac. de Ingeniería, Uruguay. \\ Sylvie Oliffson Kamphorst ${ }^{\dagger}$ \\ Sônia Pinto de Carvalho \\ Departamento de Matemática, ICEx, UFMG, Brasil.
}

\begin{abstract}
The elliptical stadium is a curve constructed by joining two half-ellipses, with half axes $a>1$ and $b=1$, by two straight segments of equal length $2 h$.

Donnay [6] has shown that if $1<a<\sqrt{2}$ and if $h$ is big enough, then the corresponding billiard map has a positive Lyapunov exponent almost everywhere; moreover, $h \rightarrow \infty$ as $a \rightarrow \sqrt{2}$.

In this work we prove that if $1<a<\sqrt{4-2 \sqrt{2}}$, then $h>2 a^{2} \sqrt{a^{2}-1}$ assures the positiveness of a Lyapunov exponent. And we conclude that, for these values of $a$ and $h$, the elliptical stadium billiard mapping is ergodic and has the K-property.
\end{abstract}

\section{Introduction}

The plane billiard consists in the free motion of a point particle on a connected bounded region in $\mathcal{R}^{2}$, being reflected elastically at the boundary. The billiard defines a 2-dimensional discrete dynamical system.

Depending on the boundary, this discrete dynamical system may have different dynamical behaviour. For instance, if it is a circle or an ellipse, it is known that the system is integrable, and the phase space is ordered by invariant curves (figure 1).

A quite different situation appears when the components of the boundary have negative curvature. The system is, then, ergodic and almost every orbit is dense on the phase space (as in Sinai billiards).

The first example of an ergodic billiard with convex boundary was given by Bunimovich [3]. The boundary is the circular stadium, composed by joining two half-circles by means of two straight segments of equal length.

A convex generalization is the elliptical stadium, composed by two half-ellipses, with half axes $a \geq 1$ and $b=1$, joined by two straight segments of equal length $2 h$. For $a=1$, ie, for the circular stadium billiard, $h>0$ implies the existence of a positive Lyapunov exponent almost everywhere. This will not be the case for $a \neq 1$ and small values of $h$, because the structure of the phase space of the elliptical billiard is not as degenerate as that of the circular billiard (figure 1).

\footnotetext{
*During this work, partially supported by Fac. de Ciencias, Uruguay.

$\dagger$ Partially supported by CNPq, Brasil.
} 

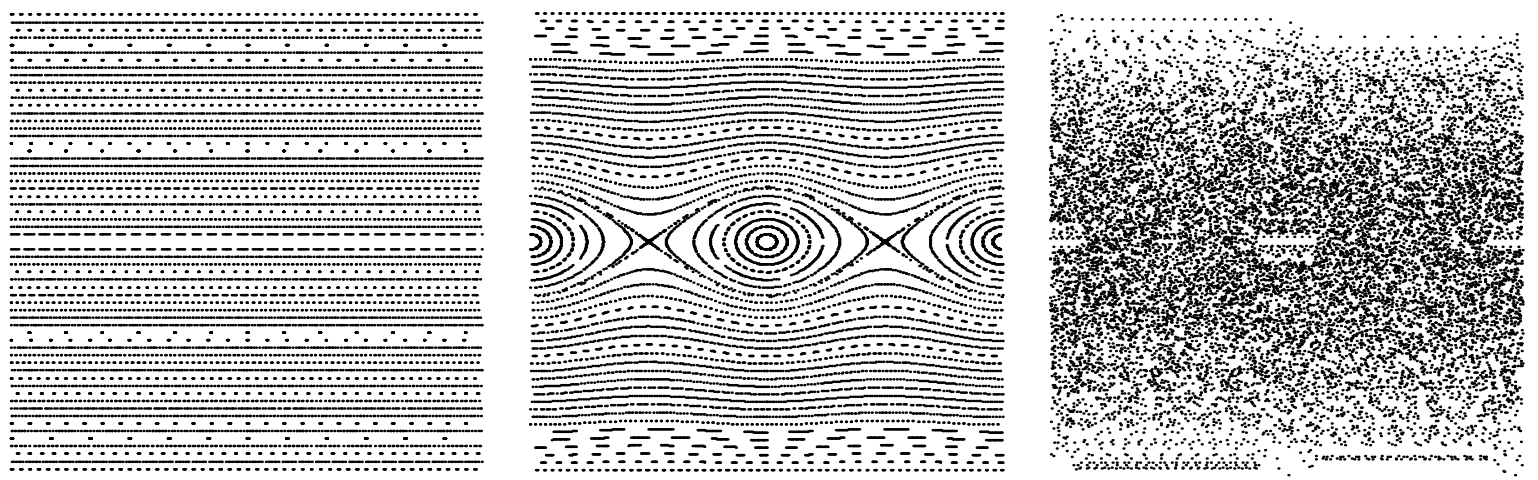

Figure 1: Phase space of the circular, the elliptical and the circular stadium billiards

V. Donnay, in [6], proved that for the billiard on the elliptical stadium, if $1<a<\sqrt{2}$ and $h$ is sufficiently $\mathrm{big}$, then there is a positive Lyapunov exponent almost everywhere. Also that $h$ must grow as $a$ increases to $\sqrt{2}$. And he gives a challenge: "One could try to calculate bounds on these lengths."

In 4 , Canale and Markarian studied this problem numerically and, varying $a$, found a value $h(a)$ such that if $0 \leq h<h(a)$, the billiard does not seem to be chaotic and, if $h>h(a)$, it seems ergodic.

In this article we give a partial answer to Donnay's challenge. We prove that if $1<a<\sqrt{4-2 \sqrt{2}}$, then $h>2 a^{2} \sqrt{a^{2}-1}$ assures the positiveness of a Lyapunov exponent. To do so, we construct a cone-field, eventually strictly invariant under the derivative of the billiard map, and apply Wojtkowski's Theorem 114. The magic number $\sqrt{4-2 \sqrt{2}}$ is related to the structure of bifurcation of hyperbolic caustic periodic orbits of the elliptical billiard (see figure 3).

The existence of a positive Lyapunov exponent, meaning sensitive dependence on initial conditions, does not automatically imply ergodicity. However, in this example, as the numerical experiments suggest, ergodicity (and K-property) can be proved. In fact, the additional conditions required on the Fundamental Theorem of Sinai and Chernov are satisfied by this system (see [10] and [8]).

\section{Billiards}

Let $\Gamma$ be an oriented plane closed curve, or a finite set of (topologically) closed curves on the plane, and $Q$ the region enclosed by $\Gamma$. The billiard problem consists in the free motion of a point particle on $Q$, being reflected elastically at the impacts on $\Gamma$. At any instant, the state of the particle is determined by its position and velocity. Since the motion is free on $Q$, all the motion is determined either by two consecutive points of reflection at $\Gamma$ or by the point of reflection and the direction of motion immediately after each reflection.

Let $t \in[0, L)$ be a parameter for $\Gamma$ and the direction of motion be determined by the angle $\theta$ with the tangent to the boundary. The billiard defines a map $T$ from the annulus $\mathcal{A}=[0, L) \times(0, \pi)$ into itself. Let $\left(t_{0}, \theta_{0}\right)$ and $\left(t_{1}, \theta_{1}\right) \in \mathcal{A}$ be such that $T\left(t_{0}, \theta_{0}\right)=\left(t_{1}, \theta_{1}\right)$. As usual, we will call $t$ either the value of the parameter or the point $\Gamma(t)$ at the boundary. If $\Gamma$ is $C^{k}, k \geq 2$, in some neighborhoods of $t_{0}$ and $t_{1}$, then $T$ is a $C^{k-1}$-diffeomorphism in some neighborhoods of $\left(t_{0}, \theta_{0}\right)$ and $\left(t_{1}, \theta_{1}\right)$ [13]. $T$ also preserves the measure $d \mu=\sin \theta d \theta \frac{d s}{d t} d t$ [2], where $s$ is the arclenght parameter for $\Gamma$.

$(\mathcal{A}, \mu, T)$ defines a discrete dynamical system, whose orbits are given by $\left\{\left(t_{n}, \theta_{n}\right)=T^{n}\left(t_{0}, \theta_{0}\right), n \in Z\right\}$.

The polygonal on $Q$, whose vertices are $\Gamma\left(t_{n}\right), n \in Z$, is often called the trajectory of the particle. Because of the obvious correspondence between orbits and trajectories, we will call both of them either orbits or trajectories. 


$\varphi_{2} \quad \begin{array}{llll} & \varphi_{1} & & \\ & \theta_{1} & & \\ & & & \\ & & \theta_{0} & \varphi_{0}\end{array}$

Figure 2: Coordinates for the elliptical billiard

\subsection{The Elliptical Billiard}

Given an orthogonal coordinate system in $\mathcal{R}^{2}$, let $\Gamma$ be the ellipse $\frac{x^{2}}{a^{2}}+y^{2}=1$, with $a>1$. We will use the angle $\varphi$ between the oriented tangent and the $x$-axis as a parameter for $\Gamma$ (figure 2). We will call half-ellipses the pieces of $\Gamma$ corresponding to $0 \leq \varphi \leq \pi$ and $\pi \leq \varphi \leq 2 \pi$.

Each trajectory has a conic caustic, confocal with $\Gamma$. If a segment of a given trajectory cuts the segment joining the two foci, all the other segments of this trajectory will cut it and the caustic will be a hyperbola. If it passes by one focus, the trajectory will always pass by the foci, thus having the two foci as a degenerate caustic. Otherwise, the caustic will be an ellipse (see, for instance [5]).

The phase space of the elliptical billiard is the annulus $\mathcal{A}=[0,2 \pi) \times(0, \pi)$, with coordinates $\varphi$ and $\theta$. As $\Gamma$ is $C^{\infty}$, the map $T: \mathcal{A} \rightarrow \mathcal{A}$ is a $C^{\infty}$-diffeomorphism, where $\left(\varphi_{1}, \theta_{1}\right)=T\left(\varphi_{0}, \theta_{0}\right)$ is given by:

$$
\begin{aligned}
a \tan \varphi_{1} & =\frac{a \tan \gamma-a \tan \varphi_{0}}{1+(a \tan \gamma)\left(a \tan \varphi_{0}\right)}, a \tan \gamma=\frac{2 a \tan \left(\varphi_{0}+\theta_{0}\right)}{1-\left(a \tan \left(\varphi_{0}+\theta_{0}\right)\right)^{2}} \\
\theta_{1} & =\varphi_{1}-\left(\varphi_{0}+\theta_{0}\right)
\end{aligned}
$$

and the derivative of $T$ is (see, for instance [13]):

$$
D T_{\left(\varphi_{0}, \theta_{0}\right)}=\frac{1}{R_{1} \sin \theta_{1}}\left(\begin{array}{cc}
l_{01}-R_{0} \sin \theta_{0} & l_{01} \\
l_{01}-R_{0} \sin \theta_{0}-R_{1} \sin \theta_{1} & l_{01}-R_{1} \sin \theta_{1}
\end{array}\right),
$$

where $l_{01}$ is the distance between $\left(x\left(\varphi_{0}\right), y\left(\varphi_{0}\right)\right)$ and $\left(x\left(\varphi_{1}\right), y\left(\varphi_{1}\right)\right)$, and $R_{i}=a^{2} /\left(a^{2} \sin ^{2} \varphi_{i}+\cos ^{2} \varphi_{i}\right)^{3 / 2}$ is the radius of curvature at $\varphi_{i}, i=0,1$.

$T$ has no fixed points and has exactly four two-periodic orbits, being two elliptic (both eigenvalues of $D T^{2}$ have moduli one) and two hyperbolic (the moduli of the eigenvalues of $D T^{2}$ are one smaller and the other greater than one).

The function $F(\varphi, \theta)=\frac{\cos ^{2} \theta-\varepsilon^{2} \cos ^{2} \varphi}{1-\varepsilon^{2} \cos ^{2} \varphi}$, where $\varepsilon=\frac{\sqrt{a^{2}-1}}{a}$ is the eccentricity of the ellipse $\Gamma$, is a first integral for $T$, ie, $F$ is constant on orbits under $T$ (see, for instance, [7]). Physically, $F$ may be interpreted as the product of angular momenta about the two foci [1]. Geometrically, $\sqrt{\left|F\left(\varphi_{0}, \theta_{0}\right)\right|}$ measures the length of the minor axis of the conical caustic [12]. So, for every $a>1$, the phase space $\mathcal{A}$ is foliated by the level curves of $F$ (figure $\mathbb{1}$ ).

Let us call $\mathcal{E}=\{$ trajectories with elliptical caustic $\}$ and $\mathcal{H}=\{$ trajectories with hyperbolic caustic $\}$. The obvious correspondence between trajectories on the configuration space $Q$ and orbits on the phase space $\mathcal{A}$ gives the following geometrical interpretation: 
- trajectories over the major axis $\longleftrightarrow$ period 2, hyperbolic orbits, $F=0$

- trajectories over the minor axis $\longleftrightarrow$ period 2, elliptic orbits, $F=1-a^{2}$

- trajectories that pass by the foci $\longleftrightarrow$ saddle connection, $F=0$

- trajectories on $\mathcal{E} \longleftrightarrow$ orbits with $F>0$

- trajectories on $\mathcal{H} \longleftrightarrow$ orbits with $1-a^{2}<F<0$.

Because of this interpretation, we will also call $\mathcal{E}$ the set of orbits with $F>0$ and $\mathcal{H}$ the set of orbits with $1-a^{2}<F<0$.

Poncelet's theorem [5] assures that trajectories that share the same caustic have the same dynamical behaviour. And to each conical caustic, or equivalently, to each integral curve $F=k$, we can associate a rotation number (see, for instance, 11]).

- For $k>0$ (trajectories on $\mathcal{E}$ ) Jacobi coordinates (see, for instance [5]) give a natural conjugation between $\mathcal{E}$ and the trajectories of the circular billiard with $\theta \neq \pi / 2$. It follows that, to each $k>0$ we can associate a rotation number $\rho(k) \in(0,1)$, such that:

- $\rho=\frac{n}{p} \in \mathcal{Q},(n, p) \equiv 1$ corresponds to periodic orbits of period $p$, with $T^{p}\left(\varphi_{0}, \theta_{0}\right)=$ $\left(\varphi_{0}+2 n \pi, \alpha_{0}\right) \cong\left(\varphi_{0}, \alpha_{0}\right)$.

- $\rho \in \mathcal{R} \backslash \mathcal{Q}$ corresponds to dense orbits (dense on the level curve).

- For $1-a^{2}<k<0$ (trajectories on $\mathcal{H}$ ) notice that every periodic orbit has an even period and $(0, \pi / 2)$ and $(\pi, \pi / 2)$ are elliptic fixed points of the integrable, measure preserving diffeomorphism $T^{2}$, with eigenvalues

$$
\lambda_{j}=e^{2 \pi i \nu_{j}(a)}, \text { where } \nu_{j}(a)=\frac{1}{\pi} \arctan \frac{ \pm 2 \sqrt{a^{2}-1}}{2-a^{2}}, j=1,2 .
$$

Notice that for $a=\sqrt{2}, \lambda_{j}= \pm 1$.

So, to every level curve it is associated a rotation number $\tau$ such that

$$
\nu(a)=\frac{1}{\pi} \arctan \frac{2 \sqrt{a^{2}-1}}{a^{2}-2}<\tau<1 .
$$

$-\tau=\frac{n}{p} \in \mathcal{Q},(n, p) \equiv 1$, corresponds to periodic orbits of period $2 p$, crossing $2 n$ times the minor axis of $\Gamma$.

$-\tau \in \mathcal{R} \backslash \mathcal{Q}$ corresponds to dense orbits (dense on the level curve).

Notice that $\nu(a)$ is strictly decreasing, $\nu(a) \longrightarrow 1$, as $a \longrightarrow 1^{+}$and $\nu(a) \longrightarrow 0^{+}$, as $a \longrightarrow+\infty$. This indicates the first difference between the trajectories on $\mathcal{E}$ and on $\mathcal{H}$ : for each $a>1$, we have periodic orbits of every period on $\mathcal{E}$, but not on $\mathcal{H}$. For instance, orbits of period 4 exist only for $a>\sqrt{2}$, or there is no period 8 orbits if $a<\sqrt{4-2 \sqrt{2}}$. On figure 3, we show the bifurcation diagram for periodic orbits on $\mathcal{H}$.

Another difference lies on where the trajectory touches the conical caustic. The tangency to the caustic on $\mathcal{E}$ always occurs inside $Q$ (ie, inside the original ellipse) and so, inside the segment of trajectory that joins two reflections at $\Gamma$. However, on $\mathcal{H}$, the tangency may occur outside $Q$, ie, you do not necessarily have the point of tangency between two reflections at $\Gamma$. It may occurs even at infinity, if a segment of trajectory is contained on an asymptote of the caustic hyperbola.

The results below give conditions to allow tangencies on $Q$ for trajectories on $\mathcal{H}$. 


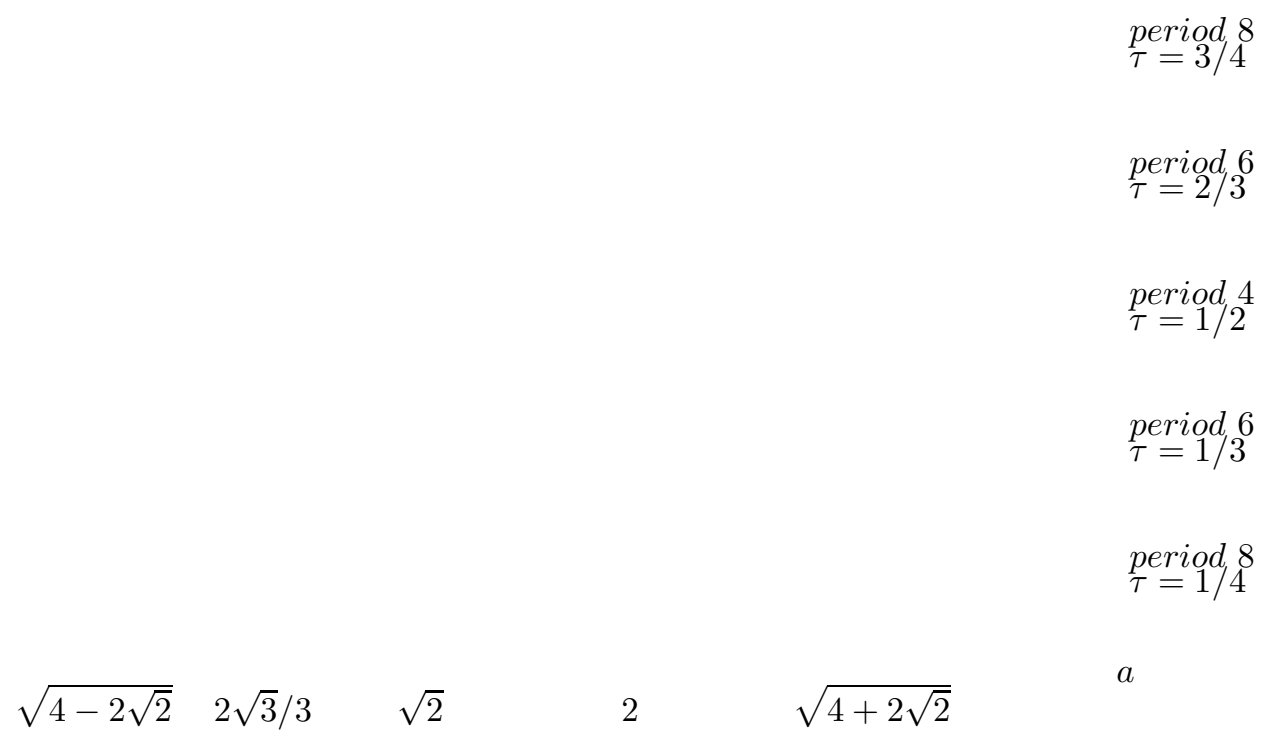

Figure 3: Diagram of bifurcation of periodic orbits on $\mathcal{H}$

Lemma 1 Suppose that a trajectory $\left\{T^{n}\left(\varphi_{0}, \theta_{0}\right)\right\} \in \mathcal{H}$ has two consecutive reflections at the same halfellipse. If $1<a<\sqrt{2}$, then the tangency between the segment joining those two reflections and the hyperbolic caustic occurs inside the billiard.

proof: Suppose that those two consecutive reflections are at $\varphi_{0} \in[0, \pi / 2]$ and $\varphi_{1} \in[\pi / 2, \pi]$ (the other cases are symmetrical). We will call $\overline{\varphi_{0} \varphi_{1}}$ the segment joining $\Gamma\left(\varphi_{0}\right)$ to $\Gamma\left(\varphi_{1}\right)$.

For a fixed $\varphi_{0}$, if $\theta_{0}=\pi / 2-\varphi_{0}$, then $\overline{\varphi_{0} \varphi_{1}}$ touches the caustic at its vertex (and so, inside $Q$ ). Suppose that there is a $\theta$ such that $\varphi_{1} \in[\pi / 2, \pi]$, and $\overline{\varphi_{0} \varphi_{1}}$ touches the caustic outside $Q$. Then there is a $\tilde{\theta}_{0}$ such that $\varphi_{1} \in[\pi / 2, \pi]$, and $\overline{\varphi_{0} \varphi_{1}}$ has the tangency exactly at the boundary. But this means that either $\Gamma\left(\varphi_{0}\right)$ or $\Gamma\left(\varphi_{1}\right)$ is the intersection of the hyperbolic caustic and the elliptical boundary and, since they form an orthogonal family, either $\tilde{\theta}_{0}$ or $\theta_{1}=\pi / 2$.

But if $1<a<\sqrt{2}, \varphi_{0} \in[k \pi / 2,(k+1) \pi / 2]$ and $\theta_{0}=\pi / 2$, then $\varphi_{1} \in[(k+2) \pi / 2,(k+3) \pi / 2]([6])$, and this implies that $\varphi_{0}$ and $\varphi_{1}$ are not on the same half-ellipse.

Lemma 2 Suppose that a trajectory $\left\{T^{n}\left(\varphi_{0}, \theta_{0}\right)\right\} \in \mathcal{H}$ has two consecutive reflections, $\varphi_{0}$ and $\varphi_{1}$, at the same half-ellipse. If $1<a<\sqrt{4-2 \sqrt{2}}$, then the tangency between each one of the segments $\overline{\varphi_{-1} \varphi_{0}}$ and $\overline{\varphi_{0} \varphi_{1}}$ and the hyperbolic caustic occurs inside the billiard.

proof: $\left\{T^{n}\left(\varphi_{0}, \theta_{0}\right)\right\} \in \mathcal{H}$. So, $F\left(\varphi_{0}, \theta_{0}\right)=k_{0}<0$. Fix this hyperbolic caustic $k_{0}$. Call $\theta(\varphi)$ the solution of $F(\varphi, \theta(\varphi))=k_{0}$. The piece of trajectory $\left(\varphi_{-1}, \theta\left(\varphi_{-1}\right)\right),(0, \theta(0)),\left(\varphi_{1}, \theta\left(\varphi_{1}\right)\right)$ is such that 0 and $\varphi_{1} \in[0, \pi]$, and $\varphi_{-1}$ and $0 \in[\pi, 2 \pi]$. So, by lemma 1 , and since $\sqrt{4-2 \sqrt{2}}<\sqrt{2}, \overline{0 \varphi_{1}}$ and $\overline{\varphi_{-1} 0}$ touch the hyperbola inside $Q$. Suppose that for a given $\varphi_{0} \in[0, \pi / 2], F\left(\varphi_{0}, \theta_{0}\right)=k_{0}, \varphi_{1} \in[\pi / 2, \pi]$ and $\overline{\varphi_{-1} \varphi_{0}}$ touches the hyperbola outside $Q$. Then, there exists a $\tilde{\varphi_{0}} \in[0, \pi / 2]$, with $F\left(\tilde{\varphi_{0}}, \theta\left(\tilde{\varphi_{0}}\right)\right)=k_{0}, \varphi_{1} \in[\pi / 2, \pi]$, and $\overline{\varphi_{-1} \tilde{\varphi_{0}}}$ has the tangency exactly at the boundary. Since $\varphi_{1} \in[\pi / 2, \pi]$ and $a<\sqrt{2}, \theta\left(\varphi_{-1}\right)=\pi / 2$.

Fix now the configuration $\left(\varphi_{-1}, \pi / 2\right) \mapsto\left(\varphi_{0}, \theta_{0}\right) \mapsto\left(\varphi_{1}, \theta_{1}\right), \varphi_{-1} \in[3 \pi / 2, \pi], \varphi_{0} \in[0, \pi / 2], \varphi_{1} \in[\pi / 2, \pi]$. There exists a $k_{1}, 0>k_{1}>k_{0}$, such that $F\left(\varphi_{0}, \theta_{0}\right)=k_{1}$, and the piece of trajectory is $\left(\varphi_{-1}, \pi / 2\right) \mapsto$ 
Figure 4: Elliptical stadium

$\left(\varphi_{0}, \theta_{0}\right) \mapsto\left(\pi, \theta_{1}\right)$. Completing this trajectory, we see that it has period 8 and crosses the minor axis 6 times, or has $\tau=3 / 4$ that exists only if $a>\sqrt{4-2 \sqrt{2}}$.

Corollary 3 Suppose that a trajectory $\left\{T^{n}\left(\varphi_{0}, \theta_{0}\right)\right\} \in \mathcal{H}$ has two consecutive reflections $\varphi_{0}$ and $\varphi_{1}$ at the same half-ellipse. If $1<a<\sqrt{4-2 \sqrt{2}}$, then the tangency between each one of the segments $\overline{\varphi_{-1} \varphi_{0}}$, $\overline{\varphi_{0} \varphi_{1}}$ and $\overline{\varphi_{1} \varphi_{2}}$ and the hyperbolic caustic occurs inside the billiard.

proof: Apply lemma 2 twice.

\subsection{The Billiard on the Elliptical Stadium}

The boundary of the elliptical stadium is a curve $\Gamma$ constructed by joining two half-ellipses, with major axes $a>1$ and minor axes $b=1$, by two segments of equal length $2 h$, as shown in figure 4 .

$\Gamma$ is a Jordan curve, convex, but not strictly convex, globally $C^{1}$ but not $C^{2}$, and piecewise $C^{\infty}$. We parametrize $\Gamma$ by the angle $\varphi$ on the half-ellipses and by the arclenght parameter on the straight parts.

The billiard map associated to $\Gamma$

$$
\begin{array}{ccc}
S: \quad[0,2 \pi+4 h] \times(0, \pi) & \longrightarrow & {[0,2 \pi+4 h] \times(0, \pi)} \\
\left(t_{0}, \theta_{0}\right) & \longmapsto & \left(t_{1}, \theta_{1}\right)
\end{array}
$$

is piecewise $C^{\infty}$, but globally only $C^{0}$.

The derivative of $S$ is: if $t_{0}$ and $t_{1}$ belong to the elliptical part, then $D S_{\left(t_{0}, \theta_{0}\right)}$ is equal to (2));

if $t_{0}$ belongs to the elliptical part and $t_{1}$ to the straight part, then

$$
D S_{\left(t_{0}, \theta_{0}\right)}=\frac{1}{\sin \theta_{1}}\left(\begin{array}{cc}
k_{0} l_{01}-\sin \theta_{0} & l_{01} \\
-k_{0} \sin \theta_{1} & -\sin \theta_{1}
\end{array}\right) ;
$$

if $t_{0}$ belongs to the straight part and $t_{1}$ belongs to the elliptical part, then

$$
D S_{\left(t_{0}, \theta_{0}\right)}=\frac{1}{\sin \theta_{1}}\left(\begin{array}{cc}
-\sin \theta_{0} & l_{01} \\
-k_{1} \sin \theta_{1} & k_{1} l_{01}-\sin \theta_{1}
\end{array}\right)
$$

and if $t_{0}$ and $t_{1}$ belong to the straight part, then

$$
D S_{\left(t_{0}, \theta_{0}\right)}=\left(\begin{array}{cc}
-1 & l_{01} / \sin \theta_{1} \\
0 & -1
\end{array}\right)
$$

where $k_{i}$ is the curvature of the ellipse at $\varphi_{i}$ and $l_{01}$ is the length of the segment $\overline{t_{0} t_{1}}$. 
$\varphi_{1}$

$\begin{array}{ccccc}\varphi_{0} & \varphi_{0} & & \\ & & \\ & & & \\ & & & \\ 0 & \pi & \pi+2 h & 2 \pi+2 h & \varphi\end{array}$

Figure 5: Integral curves and caustics in the half-ellipses

It is easy to see that for every $\left(t_{0}, \theta_{0}\right)$, with $t_{0} \in(0, \pi)($ resp. $(\pi+2 h, 2 \pi+2 h))$, there exists an $n=n\left(t_{0}, \theta_{0}\right)$ such that $S^{n}\left(t_{0}, \theta_{0}\right)=\left(t_{n}, \theta_{n}\right)$, with $t_{n} \in(\pi+2 h, 2 \pi+2 h)$ (resp. $\left.(0, \pi)\right)$, ie, if we begin in a half-ellipse, we will reach the other one on a finite number of iterations.

This allows us to construct a restricted map:

$$
\begin{aligned}
& R: \quad \mathcal{B} \quad \longrightarrow \quad \mathcal{B} \\
& \left(\varphi_{0}, \theta_{0}\right) \longmapsto\left(\varphi_{1}, \theta_{1}\right)
\end{aligned}
$$

where $\mathcal{B}=\{[0, \pi] \bigcup[\pi+2 h, 2 \pi+2 h]\} \times(0, \pi), \varphi_{1}$ is the next reflection at a half-ellipse and $\theta_{1}$ is the direction of motion immediately after the reflection at $\varphi_{1}$.

Notice that while the reflections remain at the same half-ellipse, $R$ is just $T$, the diffeomorphism associated to the elliptical billiard. So, for those reflections, the orbit will stay on an integral curve of the elliptical billiard.

\section{Main Tools}

\subsection{Cone-fields and consequences}

Let $\Gamma=\{\gamma(t)=(x(t), y(t)), t \in[0, L)\}$ be a $C^{k}$-piecewise Jordan curve in $R^{2}, k \geq 1, Q$ the region of $R^{2}$ bounded by $\Gamma$ and $\mathcal{A}$ the annulus $[0, L) \times(0, \pi)$.

Let $T: \mathcal{A} \rightarrow \mathcal{A}$ be the local $C^{k-1}$ measure preserving diffeomorphism associated to the billiard on $Q$.

Definition 1 For each $(t, \theta) \in \mathcal{A}$, the Lyapunov exponents of $T$ at $(t, \theta)$ are given by:

$$
\begin{aligned}
& \lambda_{+}(t, \theta)=\lim _{n \rightarrow \infty} \frac{1}{n} \ln \left\|D T^{n}(t, \theta)\right\| \\
& \lambda_{-}(t, \theta)=-\lambda_{+}(t, \theta)
\end{aligned}
$$

Definition 2 Given two linearly independent vector fields $X_{1}(t, \theta)$ and $X_{2}(t, \theta)$ in the tangent space $T_{(t, \theta)} \mathcal{A} \approx R^{2}$, a cone in $T_{(t, \theta)} \mathcal{A}$ is defined by

$$
C(t, \theta)=\left\{r X_{1}(t, \theta)+s X_{2}(t, \theta), r . s \geq 0\right\} .
$$

Its interior is

$$
\operatorname{int}(C(t, \theta))=\left\{r X_{1}(t, \theta)+s X_{2}(t, \theta), r . s>0 \text { or } r=s=0\right\}
$$


$A$ measurable cone-field is a family of cones $\{C(t, \theta)\} \subset T A$ defined $\mu$-almost everywhere, and such that the vectors $X_{1}(t, \theta)$ and $X_{2}(t, \theta)$ vary measurably with $(t, \theta)$.

THEOREM (Wojtkowski,[14) Let $C(t, \theta)$ be a measurable cone field such that for almost every $(t, \theta)$,

$$
D T(C(t, \theta)) \subset C(T(t, \theta))
$$

and for almost every $(t, \theta)$ there exists a $k(t, \theta)$ for which

$$
D T^{k(t, \theta)}(C(t, \theta)) \subset \operatorname{int} C\left(T^{k(t, \theta)}(t, \theta)\right) .
$$

Then the Lyapunov exponents $\lambda_{+}(t, \theta)$ are positive for almost every $(t, \theta)$.

(Such a cone-field is called eventually strictly invariant.)

Our goal is to construct a measurable cone-field, eventually strictly invariant under the derivative $D S$ of the map associated to the billiard on the elliptical stadium. But $S$ is not differentiable at $t=0, \pi$, $\pi+2 h, 2 \pi+2 h$.

For $\alpha \in[0,2 \pi+4 h]$, let

$$
\begin{aligned}
W_{\alpha} & =\left\{\left(t_{0}, \theta_{0}\right) \in[0,2 \pi+4 h] \times(0, \pi) \text { such that } \exists n \in Z, S^{n}\left(t_{0}, \theta_{0}\right)=\left(\alpha, \theta_{n}\right)\right\} \\
& =\bigcup_{n \in Z} S^{n}(\{(\alpha, \theta), \theta \in(0, \pi)\})
\end{aligned}
$$

ie, the images of a vertical line on $[0,2 \pi+4 h] \times(0, \pi) . \mu\left(W_{\alpha}\right)=0$ because $\mu(\{(\alpha, \theta), \theta \in(0, \pi)\})=0$ and $S$ is measure preserving. Let $W=W_{0} \bigcup W_{\pi} \bigcup W_{\pi+2 h} \bigcup W_{2 \pi+2 h}$. Then $\mu(W)=0$ and we will work on $[0,2 \pi+4 h] \times(0, \pi) \backslash W$.

On the other hand, on the straight part of the elliptical stadium, $D S$ is just a translation and an inversion. So, it is enough to construct a cone-field strictly invariant under $D R$, the derivative of the restricted map $R$, on $\mathcal{B} \backslash(W \cap \mathcal{B})$.

Finally, if a given trajectory, between $\left(\varphi_{0}, \theta_{0}\right)$ and $\left(\varphi_{1}, \theta_{1}\right)=R\left(\varphi_{0}, \theta_{0}\right)$ hits $q$ times the straight part of the elliptical stadium, then

$$
D R_{\left(\varphi_{0}, \theta_{0}\right)}=\frac{(-1)^{q}}{R_{1} \sin \theta_{1}}\left(\begin{array}{cc}
l_{01}-R_{0} \sin \theta_{0} & l_{01} \\
l_{01}-R_{0} \sin \theta_{0}-R_{1} \sin \theta_{1} & l_{01}-R_{1} \sin \theta_{1}
\end{array}\right) .
$$

So, the image under $D R$ of a cone $C\left(\varphi_{0}, \theta_{0}\right)$ can be inverted when arriving at $\left(\varphi_{1}, \theta_{1}\right)$. But, as $-C=$ $\left\{r\left(-X_{1}\right)+s\left(-X_{2}\right), r s \geq 0\right\}=\left\{r X_{1}+s X_{2}, r s \geq 0\right\}=C$, all that will interest us is the slope of the boundaries of the cone.

\section{$3.2 \quad$ A little bit of optics}

We are going to follow Donnay ideas [6], giving an optical interpretation of the tangent space.

From now on, we are going to suppose that $\Gamma$ is strictly convex (although the elliptical stadium is not strictly convex, we are going to reduce our problem to the elliptical part of the boundary, looking only to the restricted map $R$ ). 
Definition $3 A$ pencil of rays is a $C^{1}$ curve in the phase space $\mathcal{A}$, given by $\eta:(-\epsilon, \epsilon) \longrightarrow \mathcal{A}, \eta(\sigma)=$ $(\varphi(\sigma), \theta(\sigma))$. The base point of the pencil is $\eta(0)=\left(\varphi_{0}, \theta_{0}\right)$, the span is $\eta^{\prime}(0)=(\alpha, \beta) \in T_{\left(\varphi_{0}, \theta_{0}\right)} \mathcal{A}$ and the slope is $\beta / \alpha$.

To each $\sigma \in(-\epsilon, \epsilon)$, we can associate a straight line $r(\sigma)$, passing by $\Gamma(\varphi(\sigma))$, with slope $\tan (\varphi(\sigma)+\theta(\sigma))$. The core of the pencil is the straight line $r_{0}=r(0)$. Let $P(\sigma)=r(\sigma) \bigcap r_{0}, \sigma \neq 0$. A pencil $\eta$ focus at $P_{0}=\lim _{\sigma \rightarrow 0} P(\sigma)$, if the limit exists.

If $P_{0}=(x, y)$ exists, it is given by the equations

$$
\begin{aligned}
\sec ^{2}\left(\varphi_{0}+\theta_{0}\right)\left(1+\frac{\beta}{\alpha}\right)\left(x-x_{0}\right) & =\left(\tan \left(\varphi_{0}+\theta_{0}\right) \cos \varphi_{0}-\sin \varphi_{0}\right) R_{0} \\
y-y_{0} & =\tan \left(\varphi_{0}+\theta_{0}\right)\left(x-x_{0}\right),
\end{aligned}
$$

where $\left(x_{0}, y_{0}\right)=\Gamma\left(\varphi_{0}\right), \theta_{0}=\theta(0)$ and $R_{0}$ is the radius of curvature of $\Gamma$ at $\varphi_{0}$.

So $P_{0}$ depends only on the base point $\left(\varphi_{0}, \theta_{0}\right)$ and the slope $\beta / \alpha$. From the point of view of focusing properties, we may identify pencils with the same base point and slope to either the linear pencil $\eta(\sigma)=$ $\left(\varphi_{0}+\alpha \sigma, \theta_{0}+\beta \sigma\right)$ or to the point $\left(\varphi_{0}, \theta_{0}, \alpha, \beta\right) \in T \mathcal{A}$. We can also define and calculate the focusing distance

$$
f=\operatorname{dist}\left(P_{0},\left(x_{0}, y_{0}\right)\right)=\frac{R_{0} \sin \theta_{0}}{1+\frac{\beta}{\alpha}} .
$$

Notice that $R_{0} \sin \theta_{0}$ is the length of the intersection of the core of the pencil with the half-osculating circle at $\varphi_{0}$ (the half-osculating circle is the circle with radius $R_{0} / 2$, tangent to $\Gamma$ at $\varphi_{0}$ ).

\subsection{Pencils of rays and beams of trajectories}

When we give a pencil of rays, we give a collection of initial conditions of the billiard ball problem inside $\Gamma$. So, to a pencil of rays there corresponds a beam of trajectories.

We may have to check if a given beam of trajectories $\eta(\sigma)=(\varphi(\sigma), \theta(\sigma)) \approx\left(\varphi_{0}+\alpha \sigma, \theta_{0}+\beta \sigma\right)$ focuses forward and backward, after and before the reflections at $\Gamma(\varphi(\sigma))$. The forward focusing distance is given by

$$
f_{s}=\frac{R_{0} \sin \theta_{0}}{1+\frac{\beta}{\alpha}}
$$

and the backward,

$$
f_{e}=\frac{R_{0} \sin \theta_{0}}{1-\frac{\beta}{\alpha}}
$$

because going backward is the same as going forward for the pencil

$$
\bar{\eta}(\sigma)=(\varphi(\sigma), \pi-\theta(\sigma)) \approx\left(\varphi_{0}+\alpha \sigma, \pi-\left(\theta_{0}+\beta \sigma\right)\right)=\left(\varphi_{0}+\alpha \sigma, \pi-\theta_{0}-\beta \sigma\right) .
$$

To compare the image of a pencil with another pencil, we will use Donnay's Focusing Lemma [6], rewritten as:

FOCUSING LEMMA Let $T: \mathcal{A} \rightarrow \mathcal{A}$ be the map associated to the billiard problem at $\Gamma$. Let $\left(\varphi_{0}, \theta_{0}\right)$, $\left(\varphi_{1}, \theta_{1}\right) \in \mathcal{A},\left(\varphi_{1}, \theta_{1}\right)=T\left(\varphi_{0}, \theta_{0}\right)$. Let $\eta(\sigma)$ be a pencil with base point $\left(\varphi_{0}, \theta_{0}\right)$, focusing forward at $P_{0}$ and $\xi(\sigma)$, a pencil with base point $\left(\varphi_{1}, \theta_{1}\right)$, focusing backward at $P_{1}$. If $P_{0}$ appears before $P_{1}$ when going from $\Gamma\left(\varphi_{0}\right)$ to $\Gamma\left(\varphi_{1}\right)$ along the trajectory, then

$$
\operatorname{slope}(T \circ \eta(\sigma))>\operatorname{slope}(\xi(\sigma)) .
$$




\section{Construction of a cone-field on TB}

\subsection{Zones of the phase space B}

Take $R: \mathcal{B} \rightarrow \mathcal{B}$, the restricted map defined in subsection $2.2 . \quad \mathcal{B}=\mathcal{B}_{1} \cup \mathcal{B}_{2}, \mathcal{B}_{1}=[0, \pi] \times(0, \pi)$, $\mathcal{B}_{2}=[\pi+2 h, 2 \pi+2 h] \times(0, \pi) \approx[\pi, 2 \pi] \times(0, \pi)$, each one corresponding to the reflections at one half-ellipse. Let $B=\mathcal{B} \backslash(W \cap \mathcal{B}), W$ the measure zero set defined in subsection 3.1 . $B=B_{1} \cup B_{2}$, $B_{i}=\mathcal{B}_{i} \backslash\left(W \cap \mathcal{B}_{i}\right), i=1,2$.

Let $B_{1}=U_{1} \bigcup M_{1}$ where

$$
\begin{aligned}
U_{1} & =\left\{\left(\varphi_{0}, \theta_{0}\right) \in B_{1} \text { such that } R\left(\varphi_{0}, \theta_{0}\right) \in B_{2} \text { and } R^{-1}\left(\varphi_{0}, \theta_{0}\right) \in B_{2}\right\} \\
M_{1} & =\left\{\left(\varphi_{0}, \theta_{0}\right) \in B_{1} \text { such that either } R\left(\varphi_{0}, \theta_{0}\right) \in B_{1} \text { or } R^{-1}\left(\varphi_{0}, \theta_{0}\right) \in B_{1}\right\}
\end{aligned}
$$

The boundary of $U_{1}$ is composed by two curves $\varphi \mapsto \theta_{ \pm}(\varphi)$ such that $R\left(\varphi, \theta_{+}(\varphi)\right)=\left(\pi, \theta_{1}\right)$ and $R^{-1}\left(\varphi, \theta_{-}(\varphi)\right)=\left(0, \theta_{1}\right)$ with equation:

$$
\tan \left(\varphi+\theta_{ \pm}(\varphi)\right)=\frac{\tan \varphi}{1 \mp \sqrt{1+a^{2} \tan \varphi}} .
$$

Notice that this boundary is contained on $W$.

By symmetry, we define $U_{2}$ and $M_{2}$, with $B_{2}=U_{2} \cup M_{2}$.

\subsection{Caustic, Vertical and Horizontal Pencils}

If $\left(\varphi_{0}, \theta_{0}\right) \in M_{1}$ (resp. $\left.M_{2}\right)$, there exist $m, n \in Z, m \leq 0 \leq n, m \neq n$ such that $R^{m}\left(\varphi_{0}, \theta_{0}\right), R^{m+1}\left(\varphi_{0}, \theta_{0}\right)$, $\ldots, R^{n-1}\left(\varphi_{0}, \theta_{0}\right), R^{n}\left(\varphi_{0}, \theta_{0}\right) \in M_{1}$ (resp. $\left.M_{2}\right)$ [6]. And $R^{i}=T^{i}, m \leq i \leq n$, because $M_{1} \subset B_{1}$ (resp. $\left.M_{2} \subset B_{2}\right)$. So, there is a piece of the orbit of $\left(\varphi_{0}, \theta_{0}\right)$ that behaves exactly as it would on the elliptical billiard. It stays on an integral curve $F=k$ of the elliptical billiard and this piece of trajectory has a caustic.

Definition 4 The caustic pencil at $\left(\varphi_{0}, \theta_{0}\right)$ is a curve $\eta:(-\epsilon, \epsilon) \rightarrow B_{1}, \eta(\sigma)=(\varphi(\sigma), \theta(\sigma))$ and $F(\eta(\sigma))=F\left(\varphi_{0}, \theta_{0}\right)$ or, by identification, a tangent vector at $\left(\varphi_{0}, \theta_{0}\right)$ to the integral curve $F=F\left(\varphi_{0}, \theta_{0}\right)$.

Clearly, a caustic pencil focuses forward and backward at the point of tangency of its core with the conic caustic. Also, caustic pencils are transformed by $T$ on caustic pencils.

Lemma 4 On the elliptical billiard, with $a<\sqrt{2}$, choose a trajectory on $\mathcal{E}$ such that $\varphi_{0} \in[\pi / 2, \pi]$, $\varphi_{0}+\theta_{0} \geq \pi$ and $\varphi_{1} \in[\pi, 2 \pi]$. The point $P_{0}$ of tangency of $\overline{\varphi_{0} \varphi_{1}}$ with the elliptical caustic is contained in the interior of the half-osculating circle at $\varphi=\pi$.

proof: Let us fix one elliptical caustic. To each $(\varphi, \theta), \varphi \in[\pi / 2, \pi], \varphi+\theta \geq \pi$, we associate $P(\varphi)$, the point of tangency with the caustic. Let $\tilde{\varphi}_{0}$ be such that $P\left(\tilde{\varphi}_{0}\right)$ is the upper vertex of the elliptical caustic. Ordering the elliptical caustic via the counter clockwise orientation, we have that $P\left(\tilde{\varphi}_{0}\right) \leq P(\varphi) \leq P(\pi)$. It is clear that $P\left(\tilde{\varphi}_{0}\right)$ is contained in the interior of the half-osculating circle at $\varphi=\pi$. The integral curve associated to this caustic has a minimum at $\varphi=\pi$. So the maximum of the forward focusing distance for the caustic pencil is $f_{s}=\frac{R_{\pi} \sin \theta}{1+0}=a^{2} \sin \theta$. 
Definition 5 The vertical pencil is defined by $v(\sigma)=\left(\varphi_{0}, \theta_{0}+\sigma\right)$ or by the vector $(0,1) \in T_{\left(\varphi_{0}, \theta_{0}\right)} B$.

The vertical pencil $v(\sigma)$ focuses forward and backward at $\Gamma\left(\varphi_{0}\right)$, ie, at the boundary of the billiard, since $\alpha=0, \beta=1$ and $f_{s, e}=\alpha \frac{R_{0} \sin \theta_{0}}{\alpha \pm \beta}=0$.

Lemma 5 Let $a<\sqrt{2}, h>0$ and $\left(\varphi_{1}, \theta_{1}\right)=R\left(\varphi_{0}, \theta_{0}\right)$. Let $v_{0}(\sigma)$ and $v_{1}(\sigma)$ be the vertical pencils at $\left(\varphi_{0}, \theta_{0}\right)$ and $\left(\varphi_{1}, \theta_{1}\right)$, respectively. Let $\eta(\sigma)=\left(\varphi_{1}+\alpha \sigma, \theta_{1}+\beta \sigma\right)$ be the linearization of the pencil $R\left(v_{0}(\sigma)\right)$. Then $\alpha \neq 0$ and $\frac{\beta}{\alpha}>0$.

proof: $(\alpha, \beta)=D R_{\left(\varphi_{0}, \theta_{0}\right)}(0,1)=\frac{(-1)^{n}}{R_{1} \sin \theta_{1}}\left(l_{01}, l_{01}-R_{1} \sin \theta_{1}\right)$. So, $\alpha \neq 0$ and $\frac{\beta}{\alpha}=\frac{l_{01}-R_{1} \sin \theta_{1}}{l_{01}}$.

If $a<\sqrt{2}$, the half-osculating circles of an ellipse with half axes $a$ and 1 are totally contained in the ellipse [6]. So, $l_{01}>R_{1} \sin \theta_{1}$ and $\frac{\beta}{\alpha}>0$.

Notice that, geometrically, this lemma shows that $R$ deviates the vertical to the right.

Definition 6 The horizontal pencil is defined by $h(\sigma)=\left(\varphi_{0}+\sigma, \theta_{0}\right)$ or by the vector $(1,0) \in T_{\left(\varphi_{0}, \theta_{0}\right)} B$.

The horizontal pencil focuses forward and backward at a distance $R_{0} \sin \theta_{0}$, ie, at the intersection of the core with the half-osculating circle at $\varphi_{0}$.

\subsection{A cone field on TB}

Definition 7 For each $(\varphi, \theta) \in B$, define the measurable cone field $\{C(\varphi, \theta)\} \subset T B$ by

- if $(\varphi, \theta) \in U_{1}$ or $U_{2}, C(\varphi, \theta)=\left\{r X_{v}+s X_{h}, r . s \geq 0\right\}$,

- if $(\varphi, \theta) \in M_{1}$ or $M_{2}, C(\varphi, \theta)=\left\{r X_{v}+s X_{c}, r . s \geq 0\right\}$,

where $X_{h}=(1,0)$ is the horizontal pencil at $(\varphi, \theta), X_{v}=(0,1)$ is the vertical pencil at $(\varphi, \theta)$ and $X_{c}=\left(1,-\frac{\partial F / \partial \varphi}{\partial F / \partial \theta}\right)$, where $F$ is the first integral of the elliptical billiard, is the caustic pencil at $(\varphi, \theta)$.

Remember that the elliptical stadium is defined by two parameters: $a$ and $h$. The two half-ellipses have minor axes equal to 1 and major axes equal to $a>1$, and the straight parts have length $2 h$.

Theorem 1 If $1<a<\sqrt{4-2 \sqrt{2}}$ and $h>2 a^{2} \sqrt{a^{2}-1}$, then $\{C(\varphi, \theta)\}$ is eventually strictly invariant under DR.

outline of the proof: If $\left(\varphi_{0}, \theta_{0}\right) \in M_{i}, i=1,2$, the trajectory associated to $\left(\varphi_{0}, \theta_{0}\right)$ has at least two consecutive reflections at the same half-ellipse, being $\varphi_{0}$ one of them. So, the orbit remains on the integral curve $F=F\left(\varphi_{0}, \theta_{0}\right)$ and the trajectory has, while staying on the same half-ellipse, a conic caustic. Taking

$$
M(i, \mathcal{E})=\left\{\left(\varphi_{0}, \theta_{0}\right) \text { such that the caustic is an ellipse }\right\}
$$

and

$$
M(i, \mathcal{H})=\left\{\left(\varphi_{0}, \theta_{0}\right) \text { such that the caustic is a hyperbola }\right\}
$$


we have $B_{i}=(M(i, \mathcal{E})) \bigcup(M(i, \mathcal{H})) \bigcup U_{i}, i=1,2$.

Our aim is to prove that for almost every $\left(\varphi_{0}, \theta_{0}\right) \in B$, with $\left(\varphi_{n}, \theta_{n}\right)=R^{n}\left(\varphi_{0}, \theta_{0}\right)$, we will have $D R^{n}\left(C\left(\varphi_{0}, \theta_{0}\right)\right) \subset C\left(\varphi_{n}, \theta_{n}\right)$ and, for almost every $\left(\varphi_{0}, \theta_{0}\right) \in B$, we can find a $k=k\left(\varphi_{0}, \theta_{0}\right)$ such that $D R^{k}\left(C\left(\varphi_{0}, \theta_{0}\right)\right) \subset \operatorname{int} C\left(\varphi_{k}, \theta_{k}\right)$.

The cones $C(\varphi, \theta)$ are bounded by the vertical pencil and by either the horizontal or the caustic pencil. If $a<\sqrt{2}$, the vertical pencil is deviated to the right under $D R$ (lemma 5 ). Since $\operatorname{det}\left(D R_{\left(\varphi_{0}, \theta_{0}\right)}\right)>0$, all we have to do is to compare the slope of the image of $X_{c}\left(\varphi_{0}, \theta_{0}\right)$ or $X_{h}\left(\varphi_{0}, \theta_{0}\right)$, under $D R$, with the slope of $X_{c}\left(R\left(\varphi_{0}, \theta_{0}\right)\right)$ or $X_{h}\left(R\left(\varphi_{0}, \theta_{0}\right)\right)$, depending on where $R\left(\varphi_{0}, \theta_{0}\right)$ is. We have to look for the point of tangency with a conical caustic or for the intersection with a half-osculating circle, forward or backward, in order to apply Donnay's Focusing Lemma. All the possible situations are analysed in 7 propositions in the appendix, and we conclude that if $1<a<\sqrt{4-2 \sqrt{2}}$ and $h>2 a^{2} \sqrt{a^{2}-1}$, the forward focusing point appears before the backward one.

\section{$5 \quad$ Main Results}

Take $S: \mathcal{A} \longrightarrow \mathcal{A}$ the map associated to the billiard on the elliptical stadium.

$\mathcal{A}=[0,2 \pi+4 h] \times(0, \pi)=\mathcal{B}_{1} \bigcup \mathcal{F}_{1} \bigcup \mathcal{B}_{2} \bigcup \mathcal{F}_{2}$, where: $\mathcal{B}_{1}=[0, \pi] \times(0, \pi), \mathcal{F}_{1}=[\pi+h, \pi+2 h] \times(0, \pi)$, $\mathcal{B}_{2}=[\pi+2 h, 2 \pi+2 h] \times(0, \pi)$ and $\mathcal{F}_{2}=[2 \pi+2 h, 2 \pi+4 h] \times(0, \pi)$, the $\mathcal{B}_{i}$ corresponding to the reflections at the elliptical part and the $\mathcal{F}_{i}$ corresponding to the reflections at the straight part.

Let $A=\mathcal{A} \backslash(W \cap \mathcal{A})$, where $W$ is the measure zero set defined in subsection 3.1. $A=B_{1} \bigcup F_{1} \bigcup B_{2} \bigcup F_{2}$, $B_{i}=\mathcal{B}_{i} \backslash\left(W \cap \mathcal{B}_{i}\right), i=1,2, F_{i}=\mathcal{F}_{i} \backslash\left(W \cap \mathcal{F}_{i}\right), i=1,2$.

As in subsection 4.1, let $U_{i}=\left\{\left(t_{0}, \theta_{0}\right) \in B_{i}\right.$ such that $S\left(t_{0}, \theta_{0}\right)$ and $\left.S^{-1}\left(t_{0}, \theta_{0}\right) \notin B_{i}\right\}$ and $M_{i}=\left\{\left(t_{0}, \theta_{0}\right) \in\right.$ $B_{i}$ such that either $S\left(t_{0}, \theta_{0}\right) \in B_{i}$ or $\left.S^{-1}\left(t_{0}, \theta_{0}\right) \in B_{i}\right\}$.

Corollary 6 For each $(t, \theta) \in A$, define the measurable cone field $\{C(t, \theta)\} \subset T A$ by

- if $(t, \theta) \in M_{1} \bigcup M_{2}, C(t, \theta)=\left\{r X_{v}+s X_{c}, r . s \geq 0\right\}$.

- if $(t, \theta) \in U_{1} \cup U_{2}, C(t, \theta)=\left\{r X_{v}+s X_{h}, r . s \geq 0\right\}$.

- if $(t, \theta) \in F_{1} \bigcup F_{2}, C(t, \theta)=D S\left(C\left(S^{-1}(t, \theta)\right)\right)$.

If $1<a<\sqrt{4-2 \sqrt{2}}$ and $h>2 a^{2} \sqrt{a^{2}-1}$, then $\{C(t, \theta)\}$ is eventually strictly invariant under DS.

Applying Wojtkowski's theorem, it follows that for those values of $a$ and $h$, the Lyapunov exponents are non-vanishing $\mu$-almost everywhere. In $([\mathbb{8}], \S 14$. A), Liverani and Wojtkowski proved that the strictly invariance of the cone-field implies the non-contraction property of its vectors. And also that the other conditions on the dynamical behaviour of the singularity curves (the vertical lines on the phase space, given by $W_{0}, W_{\pi}, W_{\pi+2 h}$ and $W_{2 \pi+2 h}$ ) have been satisfied. All those additional conditions allow to prove that the system is not only chaotic, ie, has non-vanishing Lyapunov exponents, but also ergodic and (see [10], sec. 4) is a K-system.

So, we have:

Theorem 2 If $a<\sqrt{4-2 \sqrt{2}}$, then $\forall h>2 a^{2} \sqrt{a^{2}-1}$, the map associated to the elliptical stadium has non-vanishing Lyapunov exponents $\mu$-almost everywhere, is ergodic and has the K-property. 

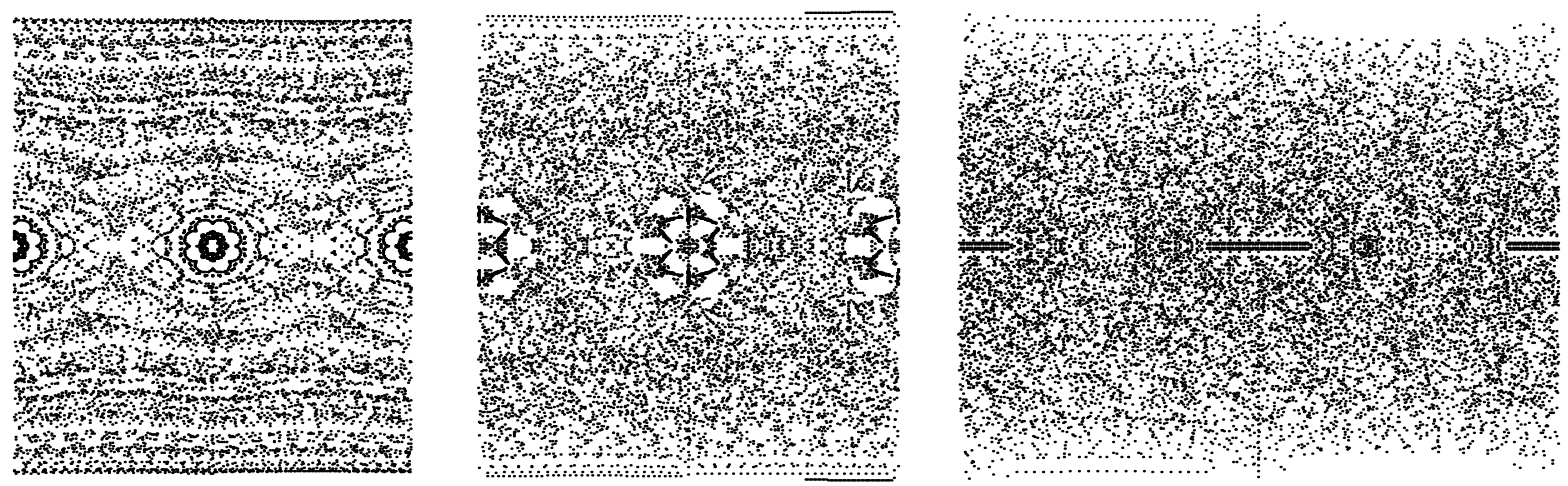

Figure 6: $a=1.07$ and $h=0.01, h=0.1, h=0.815$

\section{$6 \quad$ Final Remarks}

Actually, the numerical simulations suggest much more about the dynamical behaviour, as we present bellow.

1) $2 a^{2} \sqrt{a^{2}-1}$ does not seem to be an optimal lower bound for $h$. The phase space seems "chaotic" for values of $h$ smaller than this bound, at least for $a<\sqrt{4-2 \sqrt{2}}$.

The dependence on $\sqrt{a^{2}-1}$ seems reasonable, since it measures the focal distance of the half ellipses. What seems to be unnecessarily big is the factor $2 a^{2}$ that is clearly an overestimation for $h$, used on the proof of proposition 9, to assure that the focusing points of orbits from $M(1, \mathcal{H})$ to $M(2, \mathcal{H})$ are separated by a reflection at the straight part.

In any case, the lower bound curve $h(a)=2 a^{2} \sqrt{a^{2}-1}$ is in agreement with what we expected for $a \sim 1$. For the circular stadium billiard, or $a=1, h>0$ implies ergodicity. This will not be the case for $a \neq 1$, because the structure of the phase space of the elliptical billiard is not as degenerate as that of the circular billiard. The vertical tangent at $a=1$ reflects this fact.

2) $\forall a>1$ and $\forall h>0$, it seems that a neighbourhood of the boundaries $\theta=0$ and $\theta=\pi$ is invariant. As shown in proposition 8 , the behaviour of the cone field there is analogous to the behaviour of the cone field constructed by Donnay [6] for the circular stadium. And this, perhaps, invariant neighbourhood is a chaotic zone of positive measure, even for small $h$ 's (figure 7 ).

3) $\forall a>1$ and $\forall h>0$, some initial conditions near $\theta=0$ ( or $\theta=\pi$ ), after a finite number of iterations, come near $\theta=\pi$ (resp. $\theta=0$ ), showing that the trajectory changes orientation in relation to the orientation of the boundary of the billiard. This indicates that the invariant manifolds of the hyperbolic 2 -periodic orbit $\{(\pi / 2, \pi / 2),(3 \pi / 2+2 h, \pi / 2)\}$ (which have corners) cross and that there are homoclinic points and, then, non-integrability (figure 7$)$.

4) Canale and Markarian 4 have proved the existence of symmetric periodic orbits for the elliptical stadium and have pointed out that for $a>1, h>0$, there exists a 4-periodic orbit, which is elliptic if $h<\sqrt{a^{2}-1}$ and hyperbolic if $h>\sqrt{a^{2}-1}$. The numerical simulations they caried out show that, while it is elliptic, it is encircled by elliptic islands of positive measure and, fixing $a<\sqrt{2}$ and increasing $h$, those elliptic islands seem to be the last to disappear, among all those that can be observed, at least, for small values of $h$. For $a>\sqrt{2}$, the elliptic islands remain, even if $h$ is very big.

5) In the construction of the cone field, as in Donnay's proof [6], it becomes clear why the lower bound for $h$ must increase to infinity as $a$ approaches $\sqrt{2}$. As $a$ approaches $\sqrt{2}^{+}$, there is a trajectory on $M \cap \mathcal{H}$ 

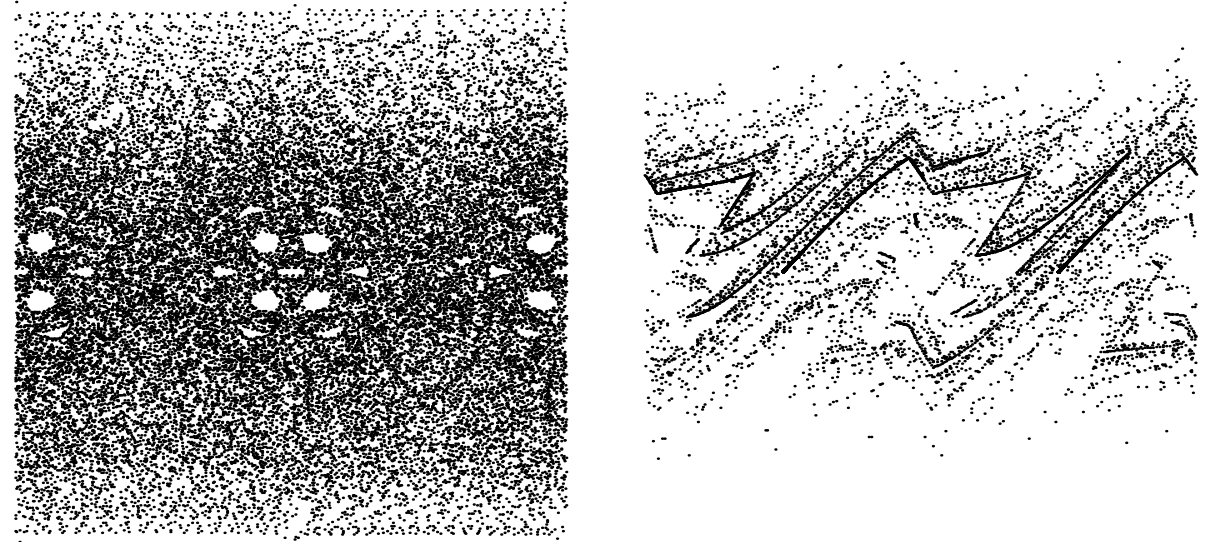

Figure 7: 32,000 points of a unique orbit and the unstable manifold, for $\mathrm{a}=1.07$ and $\mathrm{h}=0.15$

that has two reflections at the same half-ellipse and cuts the minor axis of the half-ellipse closer and closer to the center of the half-ellipse, ie, with a direction near the direction of the asymptote of the hyperbolic caustic. This means that the focusing distance of the caustic pencil goes to infinity and so, we need to put the half-ellipses farther and farther apart to assure that the caustic pencil will have enough space to focus and to open again.

This also shows that $h(a)=2 a^{2} \sqrt{a^{2}-1}$ can not be the lower bound for $a \sim \sqrt{2}$. $h(a)$ must be determined by pieces. We believe that the determination of $h(a)$ is closely related to the structure of bifurcation of periodic orbits with hyperbolic caustic on the elliptical billiard (figure B).

\section{Appendix}

We analyse, here, all the possibilities for a piece of orbit $\left\{\left(\varphi_{0}, \theta_{0}\right),\left(\varphi_{1}, \theta_{1}\right)\right\}$.

Proposition 7 Suppose that $\left(\varphi_{1}, \theta_{1}\right)=R\left(\varphi_{0}, \theta_{0}\right)=T\left(\varphi_{0}, \theta_{0}\right)$, ie, $\varphi_{1}$ and $\varphi_{0}$ are in the same half-ellipse. If $1<a$ and $\forall h \geq 0$, then $\operatorname{DR}\left(C\left(\varphi_{0}, \theta_{0}\right)\right) \subset C\left(\varphi_{1}, \theta_{1}\right)$, but the invariance is not strict.

proof: If $\left(\varphi_{1}, \theta_{1}\right)=T\left(\varphi_{0}, \theta_{0}\right)$, then $\left(\varphi_{0}, \theta_{0}\right)$ and $\left(\varphi_{1}, \theta_{1}\right)$ are on an integral curve of the elliptical billiard and $\left(\varphi_{0}, \theta_{0}\right),\left(\varphi_{1}, \theta_{1}\right) \in M_{i}, i=1,2$. The cones are:

$$
C\left(\varphi_{j}, \theta_{j}\right)=\left\{r X_{v}\left(\varphi_{j}, \theta_{j}\right)+s X_{c}\left(\varphi_{j}, \theta_{j}\right), r . s \geq 0\right\}, j=0,1 .
$$

And $D R_{\left(\varphi_{0}, \theta_{0}\right)}\left(X_{c}\left(\varphi_{0}, \theta_{0}\right)\right)=D T_{\left(\varphi_{0}, \theta_{0}\right)}\left(X_{c}\left(\varphi_{0}, \theta_{0}\right)\right)=\lambda X_{c}\left(\varphi_{1}, \theta_{1}\right), \lambda \neq 0$.

Proposition 8 Suppose that

$\left(\varphi_{0}, \theta_{0}\right) \in M(1, \mathcal{E})($ resp. $M(2, \mathcal{E}))$ and $\left(\varphi_{1}, \theta_{1}\right) \in M(2, \mathcal{E})($ resp. $M(1, \mathcal{E}))$.

If $\forall a>1$ and $\forall h>0$, then $\operatorname{DR}\left(C\left(\varphi_{0}, \theta_{0}\right)\right) \subset \operatorname{int} C\left(\varphi_{1}, \theta_{1}\right)$.

proof: $\left(\varphi_{0}, \theta_{0}\right),\left(\varphi_{1}, \theta_{1}\right) \in M_{i}, i=1,2$. and:

$$
C\left(\varphi_{j}, \theta_{j}\right)=\left\{r X_{v}\left(\varphi_{j}, \theta_{j}\right)+s X_{c}\left(\varphi_{j}, \theta_{j}\right), r . s \geq 0\right\}, j=0,1 .
$$

The caustic pencil $X_{c}\left(\varphi_{0}, \theta_{0}\right)$ focuses forward at $P_{0}$, the point of tangency of the forward segment of the trajectory with the elliptical caustic; and the caustic pencil $X_{c}\left(\varphi_{1}, \theta_{1}\right)$ focuses backward at $P_{1}$, the point 
of tangency of the backward segment of the trajectory with a new elliptical caustic. These two elliptical caustics are no longer confocal, but have the same focusing distance $c=\sqrt{a^{2}-1}$ and their foci lie on the same straight line.

If the trajectory between $\varphi_{0}$ and $\varphi_{1}$ does not hit the straight part of the elliptical stadium, elementary geometry of ellipses plus the focusing lemma give us that:

$$
\operatorname{slope}\left(D R_{\left(\varphi_{0}, \theta_{0}\right)} X_{c}\left(\varphi_{0}, \theta_{0}\right)\right)>\operatorname{slope}\left(X_{c}\left(\varphi_{1}, \theta_{1}\right)\right) .
$$

If it hits the straight part, $P_{0}$ clearly appears before $P_{1}$ when going from $\varphi_{0}$ to $\varphi_{1}$, and the focusing lemma gives us that:

$$
\operatorname{slope}\left(D R_{\left(\varphi_{0}, \theta_{0}\right)} X_{c}\left(\varphi_{0}, \theta_{0}\right)\right)>\operatorname{slope}\left(X_{c}\left(\varphi_{1}, \theta_{1}\right)\right) .
$$

Proposition 9 Suppose that either

a) $\left(\varphi_{0}, \theta_{0}\right) \in M(1, \mathcal{E})($ resp. $M(2, \mathcal{E}))$ and $\left(\varphi_{1}, \theta_{1}\right) \in M(2, \mathcal{H})$ (resp. $\left.M(1, \mathcal{H})\right)$,

or

b) $\left(\varphi_{0}, \theta_{0}\right) \in M(1, \mathcal{H})($ resp. $M(2, \mathcal{H}))$ and $\left(\varphi_{1}, \theta_{1}\right) \in M(2, \mathcal{E})$ (resp. $\left.M(1, \mathcal{E})\right)$.

c) $\left(\varphi_{0}, \theta_{0}\right) \in M(1, \mathcal{H})($ resp. $M(2, \mathcal{H}))$ and $\left(\varphi_{1}, \theta_{1}\right) \in M(2, \mathcal{H})$ (resp. $M(1, \mathcal{H})$ ).

If $1<a<\sqrt{4-2 \sqrt{2}}$ and $h>2 a^{2} \sqrt{a^{2}-1}$, then

$$
D R_{\left(\varphi_{0}, \theta_{0}\right)}\left(C\left(\varphi_{0}, \theta_{0}\right)\right) \subset \operatorname{int} C\left(\varphi_{1}, \theta_{1}\right) .
$$

proof: of a) $\left(\varphi_{1}, \theta_{1}\right),\left(\varphi_{0}, \theta_{0}\right) \in M_{i}, i=1,2$. And

$$
C\left(\varphi_{j}, \theta_{j}\right)=\left\{r X_{v}\left(\varphi_{j}, \theta_{j}\right)+s X_{c}\left(\varphi_{j}, \theta_{j}\right), r . s \geq 0\right\}, j=0,1 .
$$

The situation is equivalent to that of the last proposition, except that now the caustic defined backward by $\left(\varphi_{1}, \theta_{1}\right)$ is a hyperbola.

The proof is based on the following results:

i) if $1<a<\sqrt{2}$ and $\left(\varphi_{1}, \theta_{1}\right) \in M(i, \mathcal{H}), i=1,2$, then this part of the trajectory has exactly two reflections at the same half-ellipse, before leaving it $([6])$.

ii) On the elliptical billiard, take a trajectory on $\mathcal{H}$ having two consecutive reflections $\varphi_{0}$ and $\varphi_{1}$ at the same half-ellipse. If $1<a<\sqrt{4-2 \sqrt{2}}$, then the caustic pencils at $\varphi_{0}$ and $\varphi_{1}$ focuses forward and backward inside the ellipse (corollary 3 ).

iii) if $h>2 a^{2} \sqrt{a^{2}-1}$, then every trajectory on $M_{i} \cap \mathcal{H}, i=1,2$ has at least one reflection at the straight part of the elliptical stadium before and after the two consecutive reflections at the same half-ellipse. (proof: We should calculate the distance $d$ from $\varphi=0$ to the reflection at the straight part of the elliptical stadium. The worst situation is that of a trajectory that leaves $\varphi=0$ in the direction of the focus. Straightforward calculations lead to $d=4 c a^{2}$. )

The interior of the elliptical stadium may be seen as the union of the interior of two ellipses, with half-axes $a$ and 1 , and the interior of a rectangle, with sides $2 h$ and 2. Notice that, if $h>\sqrt{a^{2}-1}=c$, the foci of the two ellipses are separated. Let us call $E_{1}$ and $E_{2}$ the interior of the ellipses, and Q the interior of the rectangle. Notice that the straight parts of the elliptical stadium are not contained in $E_{1} \bigcup E_{2}$.

From the results above, it is clear that the trajectory from $\varphi_{0}$ to $\varphi_{1}$ touches an elliptical caustic at $P_{0}$ in $E_{1}$ (or $E_{2}$ ), hits the straight part, and then touches a hyperbolic caustic at $P_{1}$ in $E_{2}$ (or $E_{1}$ ). 
The Focusing Lemma now gives that

$$
\operatorname{slope}\left(D R_{\left(\varphi_{0}, \theta_{0}\right)} X_{c}\left(\varphi_{0}, \theta_{0}\right)\right)>\operatorname{slope}\left(X_{c}\left(\varphi_{1}, \theta_{1}\right)\right) .
$$

the proof of b) is, by symmetry, equivalent to a); the proof of c) is analogous.

Proposition 10 Suppose that either

a) $\left(\varphi_{0}, \theta_{0}\right) \in M(1, \mathcal{E})($ resp. $M(2, \mathcal{E}))$ and $\left(\varphi_{1}, \theta_{1}\right) \in U_{2}$ (resp. $\left.U_{1}\right)$

or

b) $\left(\varphi_{0}, \theta_{0}\right) \in U_{1}$ (resp. $\left.U_{2}\right)$ and $\left(\varphi_{1}, \theta_{1}\right) \in M(2, \mathcal{E})$ (resp. $M(1, \mathcal{E})$ ).

If $1<a<\sqrt{2}$ and $h>\sqrt{a^{2}-1}$, then

$$
D R_{\left(\varphi_{0}, \theta_{0}\right)}\left(C\left(\varphi_{0}, \theta_{0}\right)\right) \subset \operatorname{int} C\left(\varphi_{1}, \theta_{1}\right)
$$

proof: (of a) $)\left(\varphi_{0}, \theta_{0}\right) \in M_{i}$, and $\left(\varphi_{1}, \theta_{1}\right) \in U_{i}, i=1,2$. So, the cones are:

$$
\begin{aligned}
& C\left(\varphi_{0}, \theta_{0}\right)=\left\{r X_{v}\left(\varphi_{0}, \theta_{0}\right)+s X_{c}\left(\varphi_{0}, \theta_{0}\right), r . s \geq 0\right\} . \\
& C\left(\varphi_{1}, \theta_{1}\right)=\left\{r X_{v}\left(\varphi_{1}, \theta_{1}\right)+s X_{h}\left(\varphi_{1}, \theta_{1}\right), r . s \geq 0\right\} .
\end{aligned}
$$

We have to compare the positions of the forward focusing point $P_{0}$ of the pencil $X_{c}\left(\varphi_{0}, \theta_{0}\right)$ to the backward focusing point $P_{1}$ of the pencil $X_{h}\left(\varphi_{1}, \theta_{1}\right) . P_{0}$ is the point of tangency with the elliptical caustic and $P_{1}$ is the intersection of the backward segment of trajectory with the half-osculating circle at $\varphi_{1}$.

If the trajectory between $\varphi_{0}$ and $\varphi_{1}$ hits the straight part of the elliptical stadium, this reflection separates $P_{0}$ and $P_{1}$, and the Focusing Lemma gives

$$
\operatorname{slope}\left(D R_{\left(\varphi_{0}, \theta_{0}\right)} X_{c}\left(\varphi_{0}, \theta_{0}\right)\right)>\operatorname{slope}\left(X_{h}\left(\varphi_{1}, \theta_{1}\right)\right) .
$$

Now suppose that it does not. Suppose also that $\varphi_{0} \in[\pi / 2, \pi)$ (the other cases being analogous by symmetry).

If $\varphi_{0}+\theta_{0} \geq \pi, P_{0}$ is contained in the interior of the half-osculating circle of $\varphi=\pi$ (lemma 4 ).

On the other hand, call $\mathcal{O}_{\varphi_{0}}$ the union of the half-osculating circles, from $\varphi=\varphi_{0}$ to $\varphi=\varphi_{0}+\pi / 2$. If $a<\sqrt{2}$ and $h>c=\sqrt{a^{2}-1}, \mathcal{O}_{\pi / 2}$ and $\mathcal{O}_{3 \pi / 2}$ are disjoint and contained in the interior of the elliptical stadium 1 . Then, if $\varphi_{0} \in[\pi / 2, \pi), \varphi_{0}+\theta_{0} \geq \pi$ and $\varphi_{1} \in[3 \pi / 2,2 \pi)$, the focusing lemma applies and

$$
\operatorname{slope}\left(D R_{\left(\varphi_{0}, \theta_{0}\right)} X_{c}\left(\varphi_{0}, \theta_{0}\right)\right)>\operatorname{slope}\left(X_{h}\left(\varphi_{1}, \theta_{1}\right)\right) \text {. }
$$

If $\varphi_{0} \in[\pi / 2, \pi), \varphi_{0}+\theta_{0} \geq \pi$ and $\varphi_{1} \in(\pi, 3 \pi / 2)$, the segment of trajectory does not cross the line containing the foci of the caustics. So, it touches a new elliptical caustic on the other half-ellipse. Besides, $X_{c}\left(\varphi_{1}, \theta_{1}\right)$ has positive slope (because the integral curves on $\mathcal{E}$ are increasing for $\varphi \in(\pi, 3 \pi / 2)$ ).

As it was shown in proposition 8 ,

$$
\operatorname{slope}\left(D R_{\left(\varphi_{0}, \theta_{0}\right)} X_{c}\left(\varphi_{0}, \theta_{0}\right)\right)>\operatorname{slope}\left(X_{c}\left(\varphi_{1}, \theta_{1}\right)\right)
$$

\footnotetext{
${ }^{1}$ Given an ellipse with half-axis 1 and $a, 1<a<\sqrt{2}$, centered at $(0,0)$, the intersection of $\mathcal{O}_{\pi / 2}$ with the $x$-axis $\subset\left[-\sqrt{a^{2}-1}, a\right]$ and of $\mathcal{O}_{3 \pi / 2}$ with the $x$-axis $\subset\left[-a, \sqrt{a^{2}-1}\right]$. So, on the elliptical stadium, if $h>\sqrt{a^{2}-1}, \mathcal{O}_{\pi / 2}$ and $\mathcal{O}_{3 \pi / 2}$ are disjoint.
} 
and

$$
\operatorname{slope}\left(X_{c}\left(\varphi_{1}, \theta_{1}\right)\right)>0=\operatorname{slope}\left(X_{h}\left(\varphi_{1}, \theta_{1}\right)\right) .
$$

If $\varphi_{0}+\theta_{0}<\pi$ and $h>c=\sqrt{a^{2}-1}, P_{0}$ is contained in the interior of the half-ellipse (ie, $E_{i} \backslash\left(E_{i} \bigcap Q\right)$ ) and $P_{1}$ is contained in $\mathcal{O}_{\pi}$. So, $\operatorname{slope}\left(X_{c}\left(\varphi_{1}, \theta_{1}\right)\right)>0=\operatorname{slope}\left(X_{h}\left(\varphi_{1}, \theta_{1}\right)\right)$.

The proof of b) is analogous to a) (by symmetry).

Proposition 11 Suppose that either

a) $\left(\varphi_{0}, \theta_{0}\right) \in M(1, \mathcal{H})$ (resp. $\left.M(2, \mathcal{H})\right)$ and $\left(\varphi_{1}, \theta_{1}\right) \in U_{2}$ (resp. $\left.U_{1}\right)$.

or

b) $\left(\varphi_{0}, \theta_{0}\right) \in U_{1}$ (resp. $\left.U_{2}\right)$ and $\left(\varphi_{1}, \theta_{1}\right) \in M(2, \mathcal{H})$ (resp. $M(1, \mathcal{H})$ ).

If $1<a<\sqrt{4-2 \sqrt{2}}$ and $h>2 a^{2} \sqrt{a^{2}-1}$, then

$$
D R_{\left(\varphi_{0}, \theta_{0}\right)}\left(C\left(\varphi_{0}, \theta_{0}\right)\right) \subset \operatorname{int} C\left(\varphi_{1}, \theta_{1}\right) .
$$

proof: (of a) the proof is analogous to the first situation in proposition 10, but notice that if $\left(\varphi_{0}, \theta_{0}\right) \in$ $M(1, \mathcal{H})($ resp. $M(2, \mathcal{H}))$ and $\left(\varphi_{1}, \theta_{1}\right) \in U_{2}$ (resp. $\left.U_{1}\right)$, if $1<a<\sqrt{4-2 \sqrt{2}}$ and $h>2 a^{2} \sqrt{a^{2}-1}$, then the trajectory touches a hyperbolic caustic at $P_{0}$ in $E_{1}$ (or $E_{2}$ ), hits the straight part, and then cuts the half-osculating circle at $\varphi_{1}$.

The proof of b) is analogous to a) (by symmetry).

Proposition 12 Suppose that

$\left(\varphi_{0}, \theta_{0}\right) \in U_{1}$ (resp. $\left.U_{2}\right)$ and $\left(\varphi_{1}, \theta_{1}\right) \in U_{2}$ (resp. $\left.U_{1}\right)$.

If $1<a<\sqrt{2}$ and $h>\sqrt{a^{2}-1}$, then $D R_{\left(\varphi_{0}, \theta_{0}\right)}\left(C\left(\varphi_{0}, \theta_{0}\right)\right) \subset \operatorname{int} C\left(\varphi_{1}, \theta_{1}\right)$

proof: The cones are:

$$
C\left(\varphi_{i}, \theta_{i}\right)=\left\{r X_{v}\left(\varphi_{i}, \theta_{i}\right)+s X_{h}\left(\varphi_{i}, \theta_{i}\right), r . s \geq 0\right\} .
$$

Suppose that $\varphi_{0} \in[\pi / 2, \pi)$ (the other cases being analogous by symmetry). The forward focusing point $P_{0}$ of $X_{h}\left(\varphi_{0}, \theta_{0}\right) \in \mathcal{O}_{\pi / 2}$.

If $\varphi_{1} \in[3 \pi / 2,2 \pi)$, the backward focusing point $P_{1}$ of $X_{h}\left(\varphi_{1}, \theta_{1}\right) \in \mathcal{O}_{3 \pi / 2}$. Since $a<\sqrt{2}$ and $h>\sqrt{a^{2}-1}$, $\mathcal{O}_{\pi / 2}$ and $\mathcal{O}_{3 \pi / 2}$ are disjoint, the result follows.

If $\varphi_{1} \in(\pi, 3 \pi / 2]$, the trajectory does not cut the line containing the foci. So, the trajectory touches backward an elliptic caustic on the other half-ellipse at $\tilde{P}_{1}$. As in proposition 10 ,

$$
\operatorname{slope}\left(D R_{\left(\varphi_{0}, \theta_{0}\right)} X_{h}\left(\varphi_{0}, \theta_{0}\right)\right)>\operatorname{slope}\left(X_{c}\left(\varphi_{1}, \theta_{1}\right)\right) .
$$

But $X_{c}\left(\varphi_{1}, \theta_{1}\right)$ has positive slope (because the integral curves are increasing for $\left.\varphi \in(\pi, 3 \pi / 2)\right)$. So

$$
\operatorname{slope}\left(D R_{\left(\varphi_{0}, \theta_{0}\right)} X_{h}\left(\varphi_{0}, \theta_{0}\right)\right)>\operatorname{slope}\left(X_{c}\left(\varphi_{1}, \theta_{1}\right)\right)>0=\operatorname{slope}\left(X_{h}\left(\varphi_{1}, \theta_{1}\right)\right) .
$$

Acknowledgements. We thank profs. Eduardo Canale (Fac. Ing. , Montevideo), Mário Jorge Carneiro, Paulo César Carrião, Susana Fornari (ICEx/UFMG) and Alain Chenciner (Univ. Paris VII) for the good ideas they gave us. We thank SBM (Sociedade Brasileira de Matemática), FAPEMIG (Fundação de Amparo à Pesquisa do Estado de Minas Gerais) and PrPq-UFMG (Pró-Reitoria de Pesquisa) for sponsoring the visit of RM to the Universidade Federal de Minas Gerais. 


\section{References}

[1] Berry, M. V. : Regularity and chaos in classical mechanics, illustrated by three deformations of a circular billiard. Eur. J. Phys 2, 91-102 (1981)

[2] Birkhoff, G. D. : Dynamical Systems. Providence, RI: A. M. S. Colloquium Publications 1966 (Original ed. 1927)

[3] Bunimovich, L. A. : On billiards close to dispersing. Math. USSR Sb. 23, 45-67 (1974)

Bunimovich, L. A. : On ergodic properties of nowhere dispersing billiards. Commun. Math. Phys. 65, 295-312 (1979)

[4] Canale, E. , Markarian, R. : Simulación de billares planos. Anales IEEE, Segundo Seminario de Informática en el Uruguay, 71-96 (1991) and private communication

[5] Chang, S-J. , Friedberg, R. : Elliptical billiards and Poncelet's theorem. J. Math. Phys. 29(7), 1537-1550 (1988)

[6] Donnay, V. J. : Using integrability to produce chaos: billiards with positive entropy. Commun. Math. Phys. 141, 225-257 (1991)

[7] Levallois, P. : Non-integrabilité des billiards définis par certaines perturbations algébriques d'une ellipse et du flot géodesique de certaines perturbations algébriques d'un ellipsoide. Thèse, Univ. Paris VII (1993)

[8] Liverani, C. , Wojtkowski, M. : Ergodicity in Hamiltonian Systems. Suny, Stony Brook, preprint (1992)

[9] Markarian, R. : Introduction to the ergodic theory of plane billiards. In: Dynamical Systems, Santiago de Chile, 1990. Harlow: Longman, 327-439 1993

[10] Markarian, R. : New ergodic billiards: exact results. Nonlinearity 6, 819-841 (1993)

[11] Palis, Jr, J. , de Melo, W. : Geometric Theory of Dynamical Systems, an introduction. Berlin, Heidelberg, New York: Springer-Verlag 1982

[12] Pimentel, F. : Pontos Heteróclinos de um bilhar Elíptico Perturbado. Dissertação de mestrado, UFMG (1993)

[13] Strelcyn, J-M. : Plane Billiards as Smooth Dynamical Systems with Singularities. In: Katok, Strelcyn, in coll. with Ledrappier, Przytycki, LNM 1222. Berlin, Heidelberg, New York: Springer-Verlag 1986

[14] Wojtkowski, M. : Invariant families of cones and Lyapunov exponents. Ergod. Th. Dynam. Sys. 5, $145-161(1985)$

ROBERTO MARKARIAN

IMERL, Facultad de Ingeniería

C.C. 30

Montevideo, Uruguay.

email: roma@imerl.edu.uy
Sylvie Oliffson Kamphorst Departamento de Matemática ICEx-UFMG, C.P. 702 30161-970 Belo Horizonte, Brasil. email: syok@mat.ufmg.br
Sônia Pinto de Carvalho Departamento de Matemática ICEx-UFMG, C.P. 702 30161-970 Belo Horizonte, Brasil. email: icedo@vm1.lcc.ufmg.br 